\title{
Genetic algorithm application for enhancing state-sensitivity partitioning
}

\begin{abstract}
Software testing is the most crucial phase in software development life cycle which intends to find faults as much as possible. Test case generation leads the research in software testing. So, many techniques were proposed for the sake of automating the test case generation process. State sensitivity partitioning is a technique that partitions the entire states of a module. The generated test cases are composed of sequences of events. However, there is an infinite set of sequences with no upper bound on the length of a sequence. Thus, a lengthy test sequence might be encountered with redundant data states, which will increase the size of test suite and, consequently, the process of testing will be ineffective. Therefore, there is a need to optimize those test cases generated by SSP. GA has been identified as the most common potential technique among several optimization techniques. Thus, GA is investigated to integrate it with the existing SSP. This paper addresses the issue on deriving the fitness function for optimizing the sequence of events produced by SSP.
\end{abstract}

Keyword: Genetic Algorithm (GA); State-Sensitivity partitioning (SSP); Test case; Sequence of events; Data state 